\title{
Variation in the relative isomer abundance of synthetic and biologically derived phosphatidylethanols and its consequences for reliable quantification
}

Marc Luginbühl ${ }^{1}$, Reuben S. E. Young ${ }^{3}$, Frederike Stöth ${ }^{2}$, Wolfgang Weinmann ${ }^{2}$, Stephen J. Blanksby $^{3}$, Stefan Gaugler ${ }^{1}$

${ }^{1}$ CAMAG, Sonnenmattstrasse 11, 4132 Muttenz, Switzerland

${ }^{2}$ Institute of Forensic Medicine Bern, University of Bern, Bühlstrasse 20, 3012 Bern, Switzerland

${ }^{3}$ Central Analytical Research Facility, Institute for Future Environments, Queensland University of Technology, Brisbane QLD 4000

Corresponding author: marc.luginbuehl@camag.com

(C) The Author(s) 2020. Published by Oxford University Press. All rights reserved. For Permissions, please email: journals.permissions@oup.com 


\begin{abstract}
Phosphatidylethanol (PEth) in human blood samples is a marker for alcohol usage. Typically, PEth is detected by reversed-phase liquid chromatography coupled with negative ion tandem mass spectrometry, investigating the fatty acyl anions released from the precursor ion upon collision-induced dissociation (CID). It has been established that in other classes of asymmetric glycerophospholipids the unimolecular fragmentation upon CID is biased depending on the relative position (known as sn-position) of each fatty acyl chain on the glycerol backbone. As such, the use of product ions in selected-reaction-monitoring (SRM) transitions could be prone to variability if more than one regioisomer is present in either the reference materials or the sample. Here, we have investigated the regioisomeric purity of three reference materials supplied by different vendors, labelled as PEth 16:0/18:1. Using CID coupled with ozoneinduced dissociation, the regioisomeric purity (\% 16:0 at $s n-1)$ was determined to be $76 \%, 80 \%$ and $99 \%$. The parallel investigation of the negative ion CID mass spectra of standards revealed differences in product ion ratios for both fatty acyl chain product ions and ketene neutral loss product ions. Furthermore, investigation of the product ion abundances in CID spectra of PEth within authentic blood samples appears to indicate a limited natural variation in isomer populations between samples, with the cannonical, PEth 16:0/18:1 (16:0 at sn-1) predominant in all cases. Different reference material isomer distributions led to variation in fully automated quantification of PEth in 56 authentic dried blood spot (DBS) samples when a single quantifier ion was used. Our results suggest caution in ensuring the regioisomeric composition of reference materials are well-matched with the authentic blood samples.
\end{abstract}

\title{
Keywords
}

Phosphatidylethanol, Dried Blood Spots, Direct Alcohol Marker, Regioisomerism 


\section{Introduction}

Phosphatidylethanol (PEth) is the most promising direct alcohol marker for application in driving aptitude assessments, the evaluation of alcohol misuse in organ donors, and alcohol withdrawal and dishabituation therapy (1-3). Within human blood, PEth 16:0/18:1 (sn-1/sn-2) is the most abundant molecular species for quantification and contains one palmitic acid chain (16:0 at $s n$ 1) and one oleic acid chain $(18: 1$ at $s n-2)(3,4)$. PEth is generally monitored by the use of tandem mass spectrometry (MS-MS) operated in negative ion electrospray selected reaction monitoring (SRM) mode. In such analysis, the two fatty acyl chain fragment ions that are released from the precursor ion upon collision-induced disscociation (CID) are monitored as quantifier and qualifier ions.

Dependent on the reference material used for the quantification of PEth in authentic samples, we observed differences $(>15 \%)$ in the final concentrations between the quantifier and qualifier transitions during routine MS-MS measurements. However, we did not observe these quantification differences in the individual calibrator and quality control samples produced by spiking the reference material to blood from teetotalers. Wozny et al. observed similar effects when quantifying phosphatidylcholine in various organ tissues (5). They reported the regioisomeric purity of reference material to be crucial for the proper quantification of substances with MS/MS, as there is a preferred (but not unique) CID fragmentation site at the $s n-2$ position of diacylglycerophospholipids. Regioisomeric mixed diacyl glycerophospholipids have the same fatty acyl composition but differ in their allocation to $s n-1$ or $s n-2$ position of the glycerol backbone (i.e., PEth 16:0(sn-1)/18:1(sn-2) and PEth 18:1(sn-1)/16:0(sn-2)). Dependent on the regioisomeric purity of the reference material, these differences can result in variations in the fragmentation pattern due to the preferred fragmentation site at the $s n-2$ position.

Furthermore, Wozny et al. found that most of the analyzed diacyl glycerophospholipids of biological origin exhibit significantly higher regioisomeric purity than synthetic lipid standards (5).

In this study we investigate these regioisomeric differences in detail for PEth 16:0/18:1 from three major PEth reference standard suppliers. We thereby study the regioisomeric purity, the product ion ratios (for both fatty acyl anion and ketene neutral loss pathways) and the actual differences in PEth concentration which are observed by analyzing authentic dried blood spot samples. Study of the regioisomeric purity of the reference material by CID coupled with ozoneinduced dissociation (OzID) allowed quantification of the distribution of the fatty acyl chains at the sn-1 and sn-2 position. A comparison of CID product ion ratios in negative ion mode permits the researcher to identify standards from different manufacturers that best match the samples. This approach for comparing standards with samples for the determination of PEth regiopurity can be completed with standard MS-MS instrumentation $(6,7)$. The analysis of authentic DBS samples provides an overview of the systematic errors which may be introduced to the quantification of PEth by differences in the regioisomeric purity of the used reference material. 


\section{Experimental}

Chemicals

The internal standard for the PEth determination, PEth 16:0/18:1- $d_{5}$, was synthesized as described elsewhere and diluted in 2-propanol (8). The same internal standard solution, with the same regioisomeric ratio, was used for all the experiments. Ammonium acetate, LiChropur was obtained from Merck (Darmstadt, Germany). 2-Propanol, acetonitrile, water, and methanol, were purchased from Carl Roth (Karlsruhe, Germany) in ultra LC-MS grade quality. PEth 16:0/18:1 reference material was obtained from Avanti Polar Lipids, Inc. (powder, sodium salt, Alabaster, USA, Lot 840514C-25MG-A-014, MLOT:5552CJA014), Cerilliant (1 mg/mL solution, tetrabutylammonium salt, Round Rock, USA, Lot FN 10161801), and Chiron SA (1 mg/mL solution, ammonium salt, Trondheim, Norway, Batch 23398). Formic acid, ULC/MS, was purchased from Biosolve BV (Valkenswaard, Netherland). BioSample TFN filter paper DBS cards from Ahlstrom were used to prepare volumetric dried blood spots (súpplied by CAMAG, Switzerland).

PEth standards from above were dried under nitrogen and shipped inter-laboratory. These samples were reconstituted using LC-MS grade methanol purchased from Fisher Scientific (Schwete, Germany). For electrospray ionization experiments, a solution of methanolic sodium acetate was used, with LC-MS grade sodium acetate being purchased from Sigma-Aldrich (Munich, Germany) and being made up with the aforementioned methanol.

\section{Determination of the regioisomeric composition of standards}

The fragmentation of lipids in negative ion mode tandem mass spectrometry has widely been used as an indication of the composition of sn-positional isomers in glycerophospholipids derived from biological extracts or synthesis. Using negative polarity ion-trap CID, Ekroos et al. were able to calibrate the product ion abundances of phosphatidylcholines against phospholipase A2 ( $\left.\mathrm{PLA}_{2}\right)$ enzyme assays, which is the gold-standard in sn-positional analysis (6). However, the relative yields of the fatty acyl product ions are known to differ greatly between the various glycerophospholipid subclasses as the charged headgroup is intimately involved in the mechanism(s) of unimolecular dissociation of the ionized lipids, including the pathways associated with the liberation of the fatty acyl anions (12). Because PEth would be subject to these same fragmentation biases, observation and quantification of the ketene loss fragments (i.e., the loss of $s n-1$ and $s n-2$ as ketenes) provides a better indication of regioisomeric purity than fatty acyl fragments, but would require calibration before quantification (vide infra).

To avoid the influence of the headgroup bias on unimolecular dissociation, a tandem MS technique known as collision-induced dissociation/ozone-induced dissociation (CID/OzID) was employed. In this technique, the glycerophospholipid is ionized as a sodiated cation and activated by CID prompting an initial loss of the functionalized-phosphate headgroup from the sn-3. This results in a common diacylglycerol-type product ion that can be directly compared between different glycerophospholipid subclasses. Subsequent activation of this product ion by OzID leads to regiospecific dissociation at the $s n-2$ position revealing the regioisomeric composition. Importantly, the removal of the headgroup in the initial activation step eliminates the need for specific headgroup calibration and thus CID/OzID can reliably quantitate the snpositional isomer composition of glycerophospholipids from any sub-class. 
The regioisomeric purity of the PEth standards was determined via MS using a modified Orbitrap Elite (Thermo Fisher Scientific, Bremen, Germany) high-resolution MS capable of ozone-induced dissociation, as outlined previously (9). Briefly, ozone was produced via a highconcentration generator (HC-30, Ozone Solutions, Hull, IA, USA) and was introduced into the helium buffer gas flow before conduction through to the high-pressure region of the linear ion trap (LIT). PEth standards were prepared at $100 \mu \mathrm{M}$ concentration in methanol before being mixed 1:1 (v/v) with $500 \mu \mathrm{M}$ methanolic sodium acetate solution and introduced using a chipbased nano-electrospray (nESI) source (TriVersa Nanomate, Advion, Ithaca, NY, USA) coupled to the MS. Positive ion mode $\mathrm{MS}^{3} \mathrm{CID} / \mathrm{OzID}$ spectra were obtained based on previously published methods $(10,11)$. In short, the PEth precursor ion $\left([\mathrm{M}+\mathrm{Na}]^{+}=m / z 725.5\right)$ was mass selected with an isolation width of 1 Th and subjected to CID using a normalised collision energy of 60 and an activation time of $1 \mathrm{~ms}$. The $\mathrm{MS}^{2}$ product ion at $\mathrm{m} / z 599$ was then reisolated (using an isolation window of $1 \mathrm{Th}$ ), and trapped for $500 \mathrm{~ms}$ in the presence of ozone with no collisional activation before product ion detection in the orbitrap (using a resolution setting of 120,000 at $\mathrm{m} / \mathrm{z} 200$ ). $\mathrm{MS}^{3}$ spectra were normalized to the total ion current before signatory CID/OzID product ions were extracted (i.e., $\mathrm{m} / z 275,291,379,395,405$ \& 421). The mean average was calculated for normalized intensity values based upon 50 individual scans; relative error was also found to be in the range of $0.5-1.5 \%$ (at a $95 \%$ confidence interval). The contribution of the cannonical isomer PEth 16:0/18:1 was calculated as a percentage from the sum of the abundance of the ions $(A)$ indicative of that isomer over the abundance of ions from both isomers (i.e., \% isomeric purity $=\left[\left(A_{275}+A_{291}+A_{379}+A_{395}\right) /\left(A_{275}+A_{291}+A_{379}+A_{395}+A_{405}+\right.\right.$ $\left.\left.\left.\mathrm{A}_{421}\right)\right] \times 100\right)$.

In parallel, negative ion MS was undertaken using a nESI source coupled to the Orbitrap Elite mass spectrometer. Given the geometry of the instrument, collision-induced dissociation was performed within the linear ion trap region (with LIT mass analysis) or the HCD cell (with FT mass analysis) of the mass spectrometer. Experiments were implemented as previously published (12), with minor variations (LIT: 1 ms activation, NCE of 45; HCD: 1 ms of activation, CE of $40 \mathrm{~V})$.

\section{Determination of the product ion ratios}

Product ion ratios with different collision energies (0-60 V) were recorded for PEth 16:0/18:1 by injecting pure reference standard solutions $(5 \mu \mathrm{L}, 30 \mathrm{ng} / \mathrm{mL}$ in 2-propanol) from the three different suppliers into the online SPE-LC-MS system. To investigate ion ratios within authentic DBS samples, 10 randomly selected samples were analyzed. Afterwards, the measured signal response (area) for the two fatty acyl chains (16:0 and 18:1) were compared. The monitored SRM transitions were $701.6 \rightarrow 255.3$ for the $16: 0$ fatty acyl chain and $701.6 \rightarrow 281.35$ for the $18: 1$ fatty acyl chain. Analyte separation and detection were performed in the CAMAG DBS laboratory (Muttenz, Switzerland) as described elsewhere, transferred and optimized for a system from Shimdazu (Kyōto, Japan), using a Shimadzu 8050 MS coupled with Nexera X2 pumps (13).

Quantification of PEth in authentic samples

A total of 56 authentic samples that were drawn for the analysis of ethanol in blood were anonymized, spotted on DBS filter paper cards, and sent to the CAMAG DBS laboratory (Muttenz, Switzerland) to perform PEth analysis. To prepare calibration samples, reference material from the three PEth reference material suppliers was spiked into the blood from a teetotaler $(240 \mu \mathrm{L}$ blood $+10 \mu \mathrm{L}$ spike solution) and pipetted on DBS cards $(20 \mu \mathrm{L})$. For the quantification, a six-point calibration from 20 to $1500 \mathrm{ng} / \mathrm{mL}$ was measured in duplicate (at the 
beginning and at the end of the run) for every reference material, together with the authentic samples. Sample concentrations above the calibration range were extrapolated. PEth 16:0/18:1 was determined by fully automated DBS extraction, using a CAMAG DBS-MS 500 autosampler (Muttenz, Switzerland). The methodic details are described elsewhere and were transferred and optimized for a system from Shimdazu (Kyōto, Japan), using a Shimadzu 8050 MS coupled with Nexera X2 pumps (13). The monitored SRM transitions were $701.6 \rightarrow 255.3$ for the $16: 0$ fatty acyl chain and $701.6 \rightarrow 281.35$ for the $18: 1$ fatty acyl chain.

\section{Results}

Determination of the regioisomeric composition of standards

As described previously $(10,11)$ and as shown in Figure 1, a sodiated lipid molecular ion is first subject to CID, causing a cyclic-acetal rearrangement of the glycerol backbone and the subsequent loss of the functionalized phosphate from the sn-3 position. Importantly, the rearrangement mechanism also forms a carbon-carbon double bond at the acetal carbon of the sn-2 branch. The re-isolation and trapping of this product ion in the presence of ozone, causes characteristic fragmentation of this double bond, leading to the neutral loss of the sn-2 substituent; this allows the sn-1-related product ions to be detected and characterized. The fragment channel specificity of CID/OzID towards the neutral loss of the sn-2 has also previously been bench-marked against $\mathrm{PLA}_{2}$ assays for validity $(10)$.

Using CID/OzID, linear ion trap-based CID (LIT) and higher-energy collision-induced dissociation (HCD), the three PEth 16:0/18:1 standards were analyzed and product ion abundances were compared to estimate the composition of $s n$-positional isomers. Table 1 presents the regiospecific sn-product ions as a relative percent of total. Error analysis of the CID/OzID approach showed error ranging between 0.5 and $1.5 \%$ at the $95 \%$ confidence interval. As discussed above, the CID/OZID product ion abundances can be treated quantitively based on prior calibration of the method against PLA ${ }_{2}$-enzyme assays $(10,11)$. These data indicate that the Cerilliant standard is regiopure PEth 16:0/18:1 (99\%)—within the uncertainty of the measurement-while the Avanti and Chiron reference materials contain 24 and $21 \%$, respectively, of the non-cannonical PEth 18:1/16:0 isomer. Data from the more widely accessible but uncalibrated negative ion CID approaches are also tabulated. Both the LIT- and HCD-activation approaches agree that examination of the ketene neutral losses is more reflective of the regioisomeric purity of a sample; however, standard CID product ions are not specific to a single regioisomer. This is most notable for the isomerically pure Cerilliant standard which upon negative ion CID (in both LIT and HCD modalities) indicates fractions of PEth 18:1/16:0 ranging from $14-23 \%$. This confirms that neither the acyl chain anion nor the ketene loss product ions are purely associated with a single regioisomer and, as such, these ions can be used to provide an indication of the dominant regioisomer in a given mixture but cannot be used as quantitative measures of regiopurity without the application of a specific PEth-based calibration.

Determination of the product ion ratios

For all of the measured samples, the signal intensity for the 18:1 fatty acyl chain was higher than the signal intensity for the 16:0 fatty acyl chain. This is expected for PEth 16:0/18:1, as $18: 1$ should be the major fatty acyl being present at the sn-2 position, as long as the regioisomeric purity is above $50 \%$. Studying the ratio between the fatty acyl chains (16:0 and 
18:1) revealed that there is a difference occurring between the investigated PEth material from different manufacturers (Figure 2). At $30 \mathrm{~V}$, close to the target CE during SRM analysis, the material from Avanti Polar Lipids Inc. showed a ratio of $47 \%$, the material from Chiron SA showed a ratio of $45 \%$, and the reference material from Cerilliant showed a ratio of $31 \%$. The analysis of 10 randomly chosen authentic DBS samples from different subjects revealed a mean ratio of $31 \% \pm 2 \%$ (range: $28-33 \%$ ) at $30 \mathrm{~V}$ and is depicted for a wide range of collision energies $(0-60 \mathrm{~V})$ in Figure 3. A comparison of the ratios at the actually relevant collision energies for the quantification of PEth based on the tuning files for the compounds $(701.6 \rightarrow 281.35$ at $32 \mathrm{~V}$ and $701.6 \rightarrow 255.3$ at $37 \mathrm{~V}$ ) revealed for all the 56 authentic samples a mean ratio of $33 \% \pm 2 \%$ (range: $29-38 \%$ ).

\section{Quantification of PEth in authentic samples}

To generate the PEth 16:0/18:1 reference standard calibration curves, fatty acyl anion signal intensities for the $\mathrm{m} / \mathrm{z} 701 \rightarrow 281$ and $701 \rightarrow 255$ SRM transitions were measured and plotted independently with concentration factors. The resulting concentrations of the calibration curves and artificially spiked quality control samples showed only minor variability within the individual reference material, independent of the chosen fatty acyl anion signal. This indicates that the signal ratios are conserved across the calibration range and either SRM transition would be suitable for absolute quantification of PEth 16:0/18:1. When 56 authentic blood samples were quantified however, a variation of up to $30 \%$ was observed between the concentrations derived from either $\mathrm{m} / \mathrm{z} 281$ and $\mathrm{m} / \mathrm{z} 255$ calibrations of the Avanti and Chiron materials. This deviation was not observed when calibrating with either SRM transition of the Cerilliant material. Further investigation of the authentic blood samples presented that the ratio between the resulting concentrations from the $\mathrm{m} / \mathrm{z} 701 \rightarrow 281$ and the $701 \rightarrow 255$ SRM fatty acyl anion signals varied by $30 \% \pm 3,26 \% \pm 4$ and $2 \% \pm 4$ from the Avanti, Chiron and Cerilliant reference standards, respectively-an ode to the regioisomeric purity of each reference standard. Overall, this indicates that, if not properly accounted for, regioisomeric impurity can significantly impact PEth quantitation methods. The box plots of Figure 4 present the implications of using isomerically impure reference standards to quantify PEth 16:0/18:1 in dried blood samples $(n=56)$. Comparison between the resulting data distributions reveals that the SRM signal chosen for quantification does not impact concentration when using isomerically pure reference standards, such as the Cerilliant standard. In contrast, using reference standards that contain large contributions of the alternate regioisomer, as is the case with the Avanti and Chiron materials, leads to significant under-representation or over-representation of PEth concentrations when only using the $\mathrm{m} / \mathrm{z} 255 \mathrm{or} / \mathrm{z} 281$, respectively. Notably, the magnitude of the positive and negative deviations (Figure 4) arising from the $\mathrm{m} / \mathrm{z} 701 \rightarrow 281$ and $701 \rightarrow 255 \mathrm{SRM}$ transitions are unequal. As a consequence, taking the mean still results in an overestimation of concentration, albeit smaller in magnitude than using the $\mathrm{m} / \mathrm{z} 281$ alone, compared to the isomerically pure standard. It remains unclear if this overestimation of the mean concentrations is caused by the regioisomeric purity of the reference material or it is just a representation of the expected variation when using reference material from different manufacturers. 


\section{Discussion}

The use of CID/OzID allowed reliable quantification of the regioisomeric composition of the reference materials obtained from different suppliers. It revealed significant variation in the contribution of the non-cannonical regiosiomer PEth 18:1/16:0 to the PEth 16:0/18:1 reference material. The variation in abundance of isomeric contributors impacts on the product ion abundances upon CID in negative ion mode and may also lead to differences in chromatographic behavior of the standards on reversed-phase columns; although the latter is difficult to establish unequivocally under these experimental conditions. Nevertheless, the variation in regioisomeric composition of the reference materials will have an impact on quantification. Similarly, the naturally occurring variation in isomer populations in biological extracts (relative to the reference materials) will also have an impact. These findings suggest that the determination of regiochemical composition of glycerophospholipid standards is an important component of developing a reliable quantitative method. The variation in isomeric make-up of the PEth standards investigated in this study indicates differing synthetic strategies between suppliers and the absence of reliable analytical approaches for reporting regiopurity both in synthetic standards and in biologically derived materials. Though rapid and robust for the determination of regiopurity in a wide range of glycerophospholipids, the CID/OzID method is not widely available. Nonetheless, testing laboratories should examine their reference materials using negative ion CID where the product ion ratios of acyl chain anions or ketene loss pathways can provide a reliable measure of differing isomeric composition. Importantly, it should be recognized, that the product ion abundance ratios are also influenced by the instrument type and experimental configuration (14). The use of reference material with a regioisomeric purity matching within the range of the regioisomeric distribution within the sample is desirable for accurate quantifications.

Very high regiosiomeric purity has previously been reported for glycerophospholipids within biological lipid extracts. For example, Wozny et al. determined a purity of $97.8 \%$ and 99.9\% for the phosphatidylcholines PC 16:0/18:1 and PC 18:0/18:1, respectively, within bovine liver extracts (5). Similarly, Kozlowski et al. found PC 16:0/18:1 to be 97\% pure within chicken egg yolk extract but, in contrast, discovered that the ratio of the isomers PC 16:0/18:1 to PC 18:1/16:0 could fluctuate between $100: 1$ up to $1: 1$ in extracts from other mammalian tissues (15). Indeed, recent experiments conducted using imaging MS in combination with CID/OzID regioisomeric analysis revealed that even adjacent tissue types can exhibit widely varying ratios of sn-positional isomers (9). While the sn-positional distribution of PEth 16:0/18:1 has not been established, it is reasonable to suggest that it may track with the ratio of PC 16:0/18:1 to PC 18:1/16:0 from which it is biologically derived. Zacek et al. used negative ion mode $\mathrm{MS}^{3}$ to investigate the isomer profile of Phosphatidylcholines in the NIST human blood plasma reference material (SRM 1950) and found the cannonical PC 16:0/18:1 to be $89 \%$ of the isomer pool (16) Overall these studies suggest the potential for wide variation in the distributions of regioisomers in biological extracts that may differ between phospholipid composition, tissue or fluid type and even between individuals. Our data, based on the analysis of PEth 16:0/18:1 from 56 samples, find the fatty acyl fragment ion abundance in a very narrow band having a mean ratio of $33 \% \pm 2 \%$ (range: $29-38 \%$ ) at the relevant CE, indicating minor differences in PEth regioisomer contributions across these individuals.

Differences in regioisomeric composition between PEth in DBS extracts and the regioisomeric composition of commercially available standards has ramifications for reliable quantification using negative ion MS. As demonstrated by the analysis of 56 authentic DBS samples, 
differences between the concentrations obtained by using the quantifier signal and the concentrations obtained by using the qualifier signal show variations based on the regioisomeric composition of the reference material. The reference material from Cerilliant, having a regioisomeric purity of $99 \%$, proved to show very low differences when quantifying authentic DBS samples using either of the acyl chain fragment ions as the quantifier signal. This suggests that the blood-derived PEth has similar regioisomeric properties as the reference material from Cerilliant. However, based on the reduced number of samples investigated, we cannot conclude that a low regioisomeric PEth variability is a general property of human blood.

\section{Conclusion}

By performing an evaluation of different PEth 16:0/18:1 reference material, we have demonstrated the impact of regioisomeric purity on the quantification of authentic DBS samples. Importantly, only using one transition fatty acyl product ion transition for the quantification of PEth in blood may introduce a significant error, when reference material with a low regioisomeric purity is used for the calibration. We hope that in the future the manufacturers are paying close attention to the regioisomeric purity of their reference material and succeed in improving the overall purity to facilitate the determination of PEth in blood.

\section{Funding}

R.S.E.Y. is grateful to the Queensland University of Technology (QUT) for the provision of a postgraduate scholarship (QUTPRA) and S.J.B. acknowledges project support from the Australian Research Council (ARC, DP190101486). Access to auxiliary expertise and instrumentation was provided through QUT's Central Analytical Research Facility hosted by the Institute for Future Environments.

\section{Ethical Statement}

The use of anonymized biological material does not fall under the scope of the Human Research Act (Art. 2 HRA) in Switzerland. Samples used during this project were collected for the determination of alcohol, based on the enforcement of the Swiss road traffic act and anonymized DBS cards were generated afterward. The DBS cards analyzed in the DBS laboratory in Muttenz could not be attributed to a specific person.

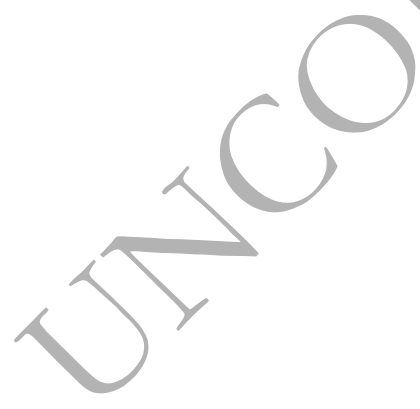




\section{References}

1. Andresen-Streichert, H., Beres, Y., Weinmann, W., Schröck, A., Müller, A., Skopp, G., et al. (2017) Improved detection of alcohol consumption using the novel marker phosphatidylethanol in the transplant setting: results of a prospective study. Transplant International, 2017: 10.1111/tri.12949.

2. Schröck, A., Pfäffli, M., König, S. and Weinmann, W. (2016) Application of phosphatidylethanol (PEth) in whole blood in comparison to ethyl glucuronide in hair (hEtG) in driving aptitude assessment (DAA). International Journal of Legal Medicine, 2016: 10.1007/s00414-016-1394-4.

3. Luginbühl, M., Weinmann, W., Butzke, I. and Pfeifer, P. (2019) Monitoring of direct alcohol markers in alcohol use disorder patients during withdrawal treatment and successive rehabilitation. Drug Testing and Analysis, 2019: 10.1002/dta.2567.

4. Gnann, H., Engelmann, C., Skopp, G., Winkler, M., Auwärter, V., Dresen, S., et al. (2010) Identification of 48 homologues of phosphatidylethanol in blood by LC-ESI-MS/MS. In Analytical and Bioanalytical Chemistry. .

5. Wozny, K., Lehmann, W.D., Wozny, M., Akbulut, B.S. and Brügger, B. (2019) A method for the quantitative determination of glycerophospholipid regioisomers by UPLC-ESI-MS/MS. Analytical and Bioanalytical Chemistry, 2019: 10.1007/s00216-018-1517-5.

6. Ekroos, K., Ejsing, C.S., Bahr, U., Karas, M., Simons, K. and Shevchenko, A. (2003) Charting molecular composition of phosphatidylcholines by fatty acid scanning and ion trap MS3 fragmentation. Journal of Lipid Research, 2003: 10.1194/jlr.D300020-JLR200.

7. Vernooij, E.A.A.M., Brouwers, J.F.H.M., Kettenes-Van Den Bosch, J.J. and Crommelin, D.J.A. (2002) RP-HPLC/ESI MS determination of acyl chain positions in phospholipids. Journal of Separation Science, 2002. 10.1002/1615-9314(20020401)25:5/6<285::AIDJSSC285>3.0.CO;2-U.

8. Luginbühl, M., Willem, S., Schürch, S. and Weinmann, W. (2018) Formation of phosphatidylethanol from endogenous phosphatidylcholines in animal tissues from pig, calf, and goat. Forensic Science International, 283, 211-218. https://www.sciencedirect.com/science/article/pii/S0379073817305467?via\%3Dihub (15 November 2019).

9. Paine, M.R.L., Poad, B.L.J., Eijkel, G.B., Marshall, D.L., Blanksby, S.J., Heeren, R.M.A., et al. (2018) Mass Spectrometry Imaging with Isomeric Resolution Enabled by Ozone-Induced Dissociation. Angewandte Chemie - International Edition, 2018: 10.1002/anie.201802937.

10. Maccarone, A.T., Duldig, J., Mitchell, T.W., Blanksby, S.J., Duchoslav, E. and Campbell, J.L. (2014) Characterization of acyl chain position in unsaturated phosphatidylcholines using differential mobility-mass spectrometry. Journal of Lipid Research, 2014: 10.1194/jlr.M046995.

11. Pham, H.T., Maccarone, A.T., Thomas, M.C., Campbell, J.L., Mitchell, T.W. and Blanksby, S.J. (2014) Structural characterization of glycerophospholipids by combinations of ozoneand collision-induced dissociation mass spectrometry: The next step towards "top-down" lipidomics. Analyst, 2014: 10.1039/c3an01712e.

12. Murphy, R.C. and Axelsen, P.H. (2011) Mass spectrometric analysis of long-chain lipids. Mass Spectrometry Reviews, 2011: 10.1002/mas.20284. 
13. Luginbühl, M., Gaugler, S. and Weinmann, W. (2019) Fully Automated Determination of Phosphatidylethanol 16:0/18:1 and 16:0/18:2 in Dried Blood Spots. Journal of Analytical Toxicology, 43, 489-496. https://academic.oup.com/jat/article/43/6/489/5486352 (15 November 2019).

14. Hou, W., Zhou, H., Khalil, M.B., Seebun, D., Bennett, S.A.L. and Figeys, D. (2011) Lysoform fragment ions facilitate the determination of stereospecificity of diacyl glycerophospholipids. Rapid Communications in Mass Spectrometry, 2011: 10.1002/rcm.4846.

15. Kozlowski, R.L., Mitchell, T.W. and Blanksby, S.J. (2015) A rapid ambient ionization-mass spectrometry approach to monitoring the relative abundance of isomeric glycerophospholipids. Scientific Reports, 2015: 10.1038/srep09243.

16. Zacek, P., Bukowski, M., Rosenberger, T.A. and Picklo, M. (2016) Quantitation of isobaric phosphatidylcholine species in human plasma using a hybrid quadrupole linear ion-trap mass spectrometer. Journal of Lipid Research, 2016: 10.1194/jlr.D070656. 
Figure Captions

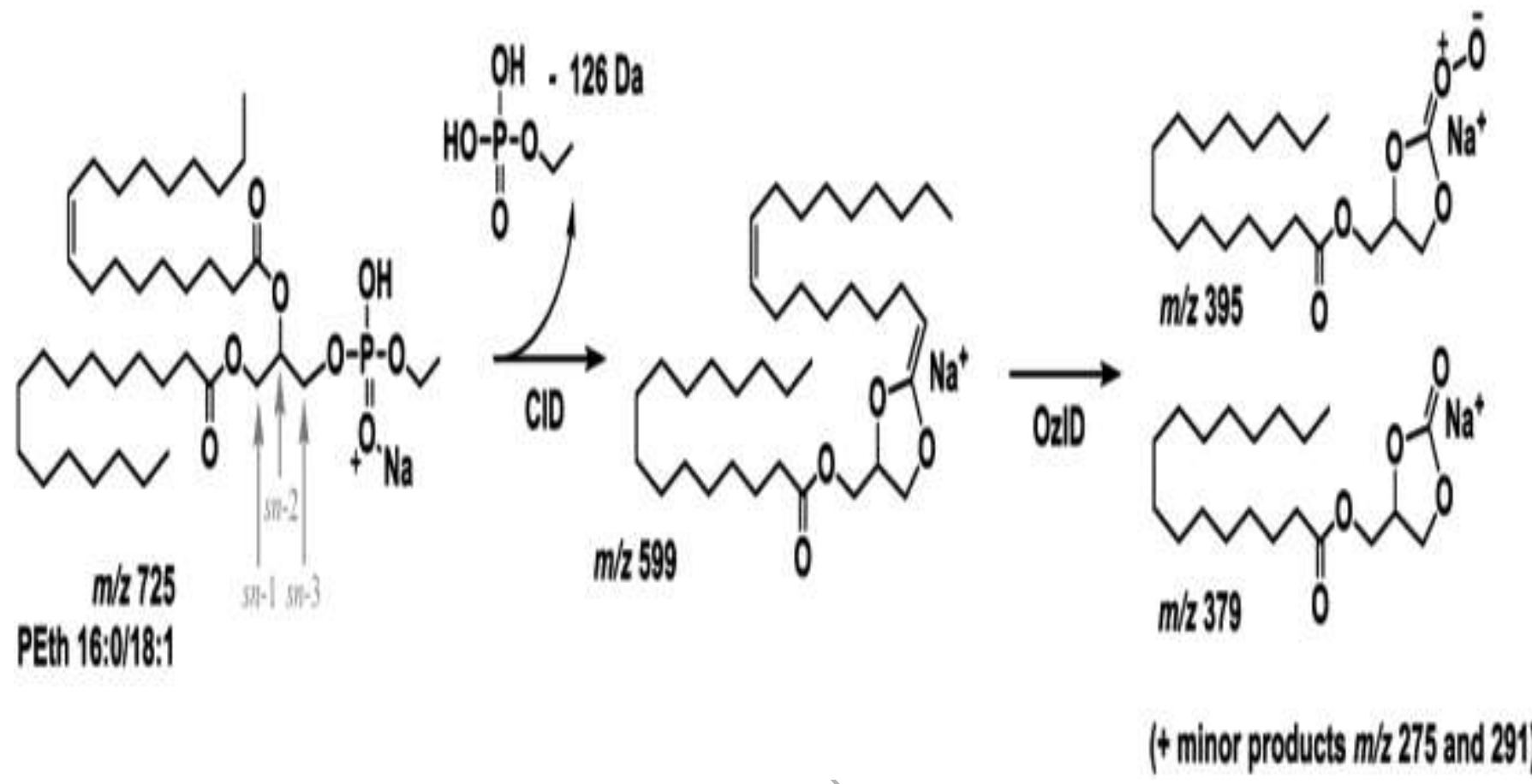

Figure 1 The CID/OzID mechanism applied to PEth 16:0/18:1. $\mathrm{MS}^{3}$ product ions show $\mathrm{m} / \mathrm{z}$ values relevant to the regioisomer with 16:0 sn-1 fatty acid. Analogous major product ions at m/z 405 and 421 are observed for the noncannonical PEth 18:1/16:0 regioisomer. 
PEth 16:0/18:1 Product Ion Ratios from Cerilliant

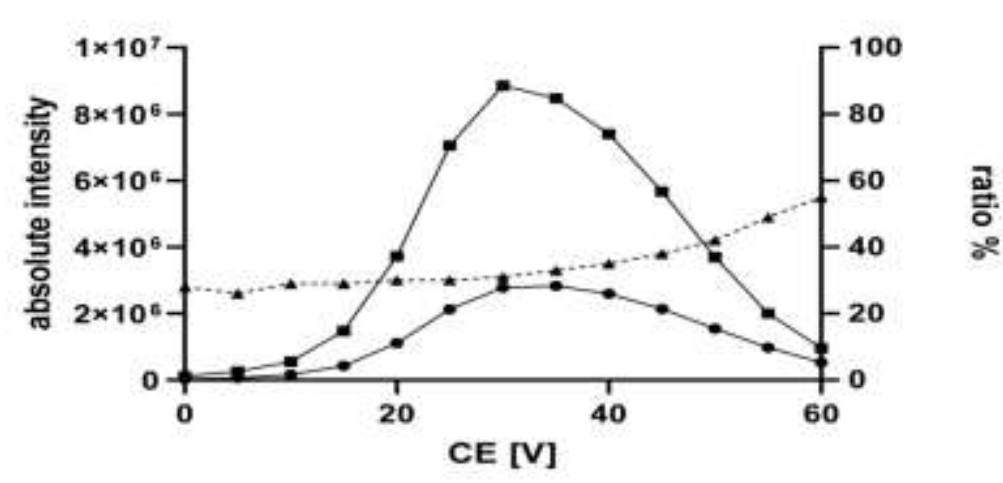

- Fatty Acyl 16:0

- Fatty Acyl 18:1

1... Ratio 16:0/18:1

PEth 16:0/18:1 Product Ion Ratios from Avanti Polar Inc

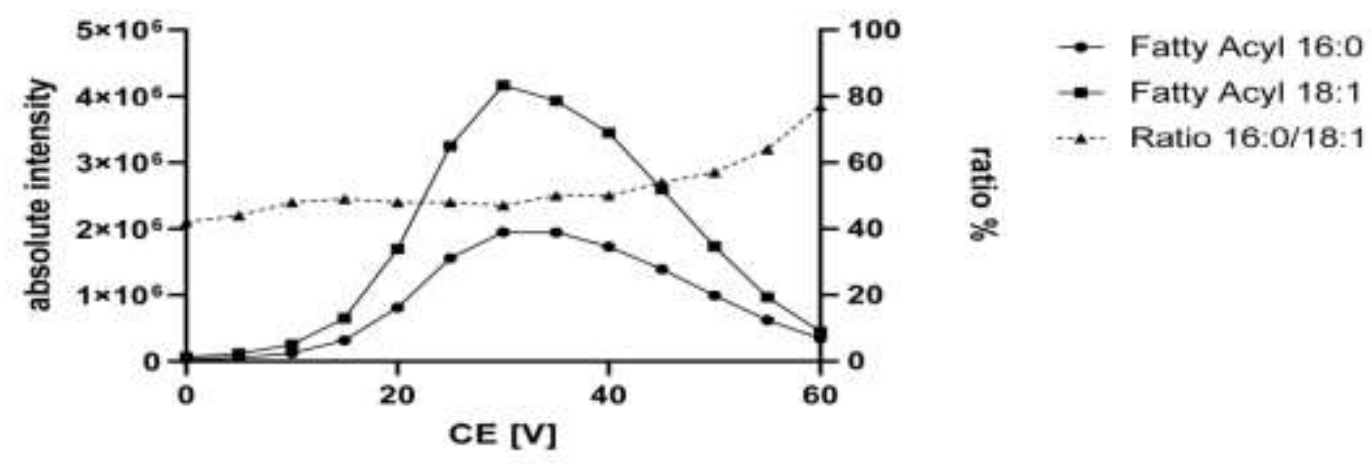

\section{PEth 16:0/18:1 Product Ion Ratios from Chiron}

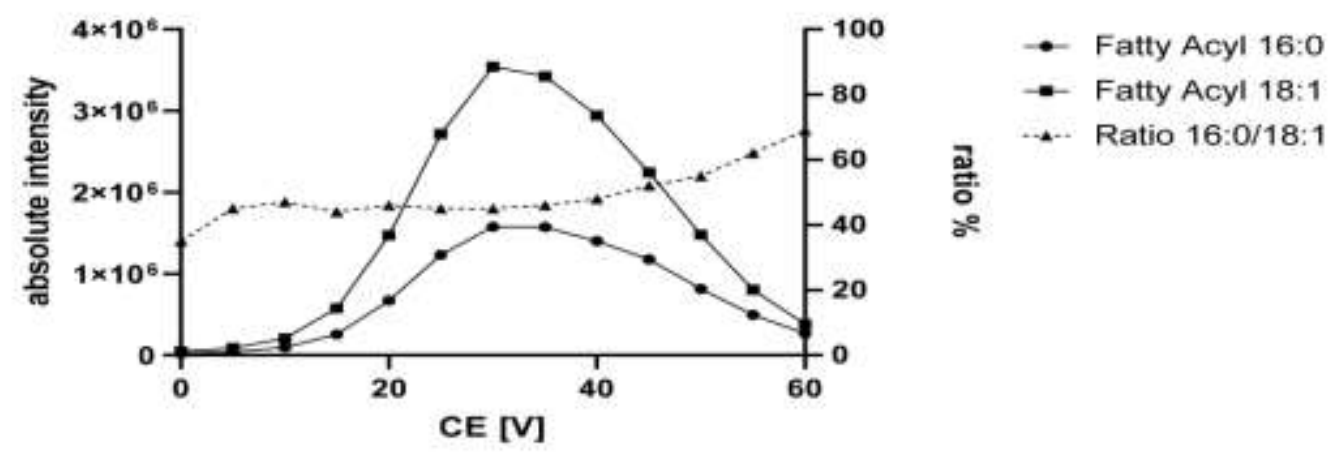

Figure 2 Product ion ratios with different collision energies (0-60 V), recorded for PEth 16:0/18:1 by injecting pure reference standard solutions (5 $\mu \mathrm{L}, 30 \mathrm{ng} / \mathrm{mL}$ in 2-propanol) from the three different suppliers into the online SPE-LCMS system 


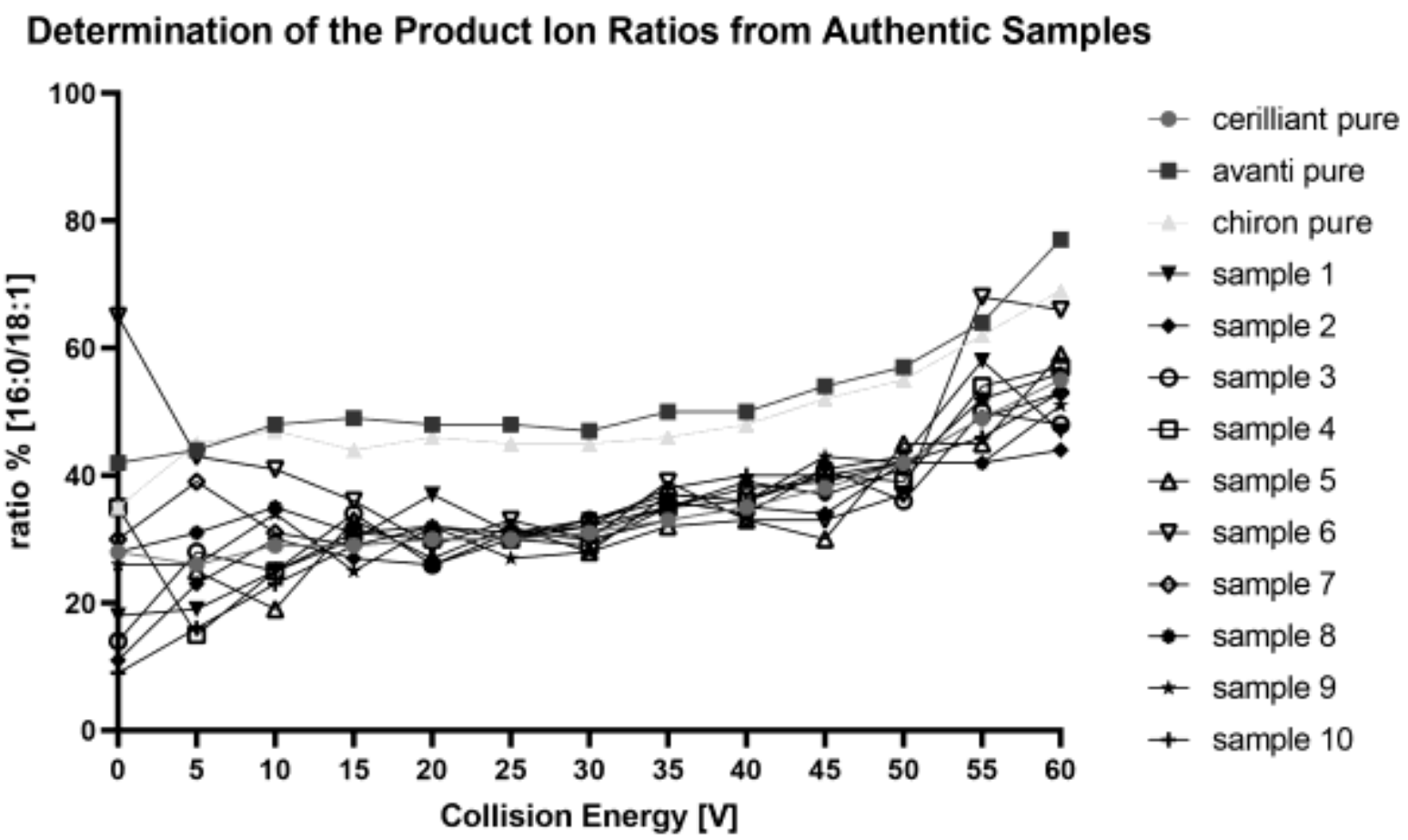

Figure 3 Product ion ratios with different collision energies (0-60 V), recorded for PEth 16:0/18:1 for 10 authentic DBS samples, compared to reference material from the three vendors. 


\section{PEth 16:0/18:1 Concentration Variance from Different Reference Standards}

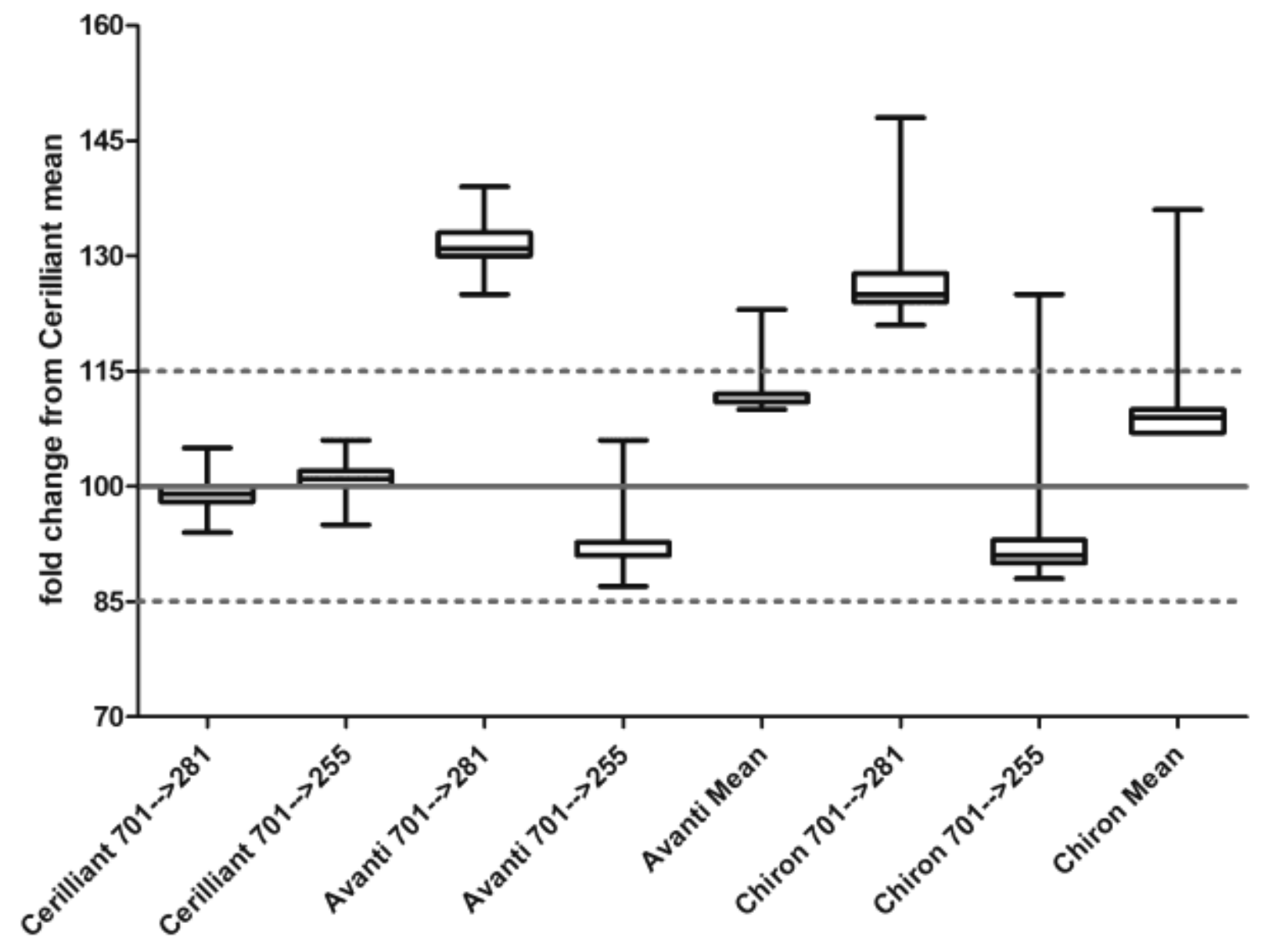

Figure 4 The comparison between the application of different reference standards for the quantitaion of PEth 16:0/18:1 in dried blood samples $(n=56)$. Labels display the fatty acyl anion SRM signals that were used for the quantification (i.e., $\mathrm{m} / \mathrm{z} 281$ or 255), normalized by the mean-averaged concentrations (i.e., $(\mathrm{m} / \mathrm{z} 281+255) / 2)$ from the isomerically pure Cerilliant reference standard. 

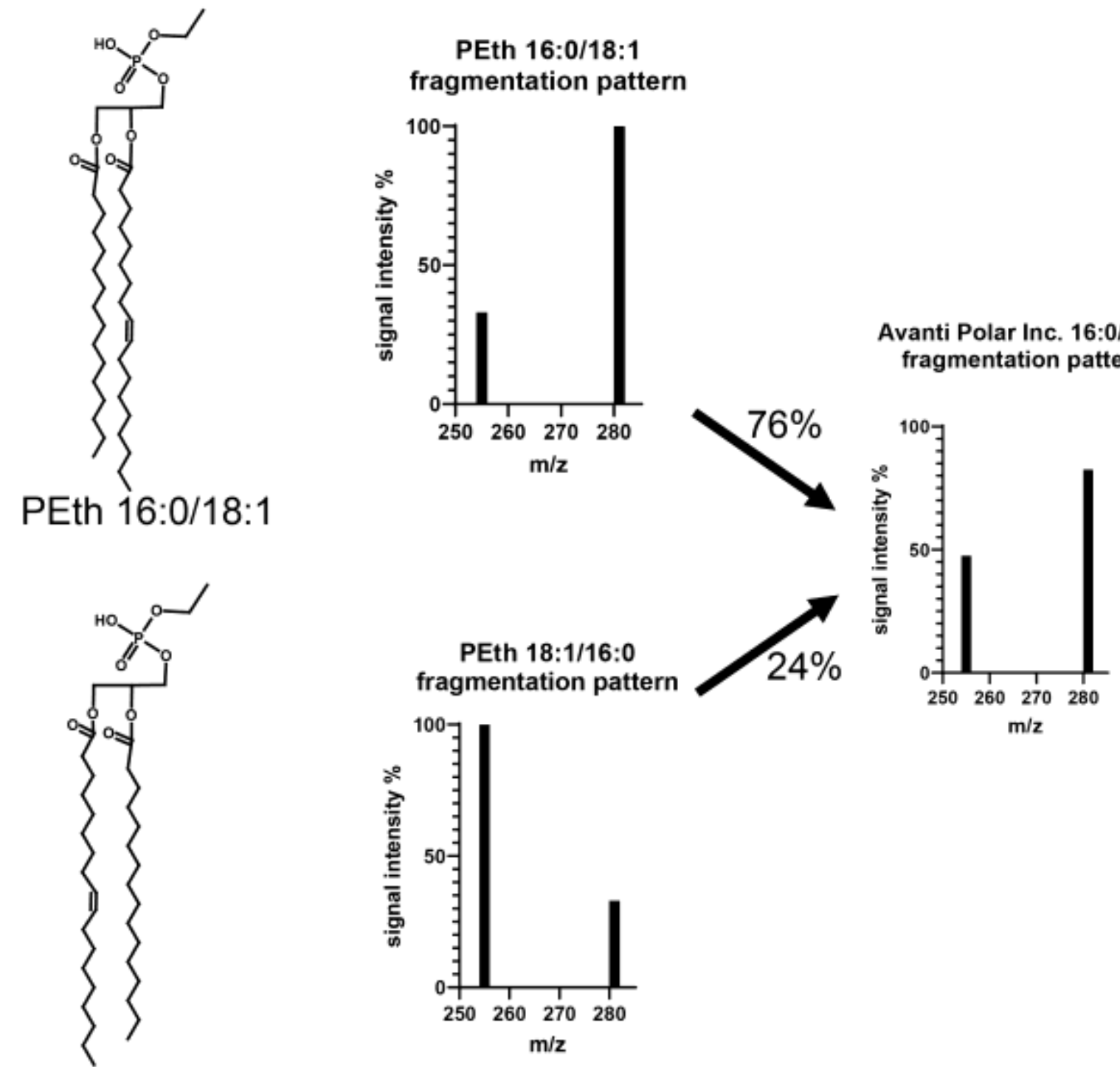

Avanti Polar Inc. 16:0/18:1 fragmentation pattern

PEth 16:0/18:1

\section{PEth 18:1/16:0}

Figure 5 Mathematical model based on experimental data collected during this study, explaining the differences in the fragmentation pattern that occur based on the regioisomeric purity. For Avanti Polar Inc. reference material, the preferred fragmentation at the sn-2 position causes the signal intensity of the quantifier $(701.6 \rightarrow 281.35)$ to decrease and the signal for the qualifier $(701.6 \rightarrow 255.3)$ to increase. As authentic blood samples demonstrated a higher regioisomeric purity, this results in an overestimation of the PEth concentration when only using the quantifier signal, and an underestimation when only using the qualifier signal during the quantification process with reference material from Avanti Polar Inc. 
Table 1. Results for the determination of the regioisomeric purity using CID/OzID, with comparisons against conventional negative ion CID methods including two different activation modes, namely, linear ion-trap (LIT) and higher-energy collision-induced dissociation (HCD)

\begin{tabular}{|c|c|c|c|c|c|c|}
\hline \multirow[b]{2}{*}{ Manufacturer } & \multirow{2}{*}{$\begin{array}{c}\text { PEth } \\
\text { 16:0/18:1 } \\
{[\% 16: 0} \\
\text { at sn-1] } \\
\text { CID/OzID } \\
(+/-1.5 \%)\end{array}$} & \multicolumn{4}{|c|}{$\begin{array}{l}\text { PEth 18:1/16:0 } \\
\text { [\% 18:1 at sn-1] }\end{array}$} & \multirow[b]{2}{*}{$\begin{array}{l}\text { HCD MS } \\
\text { (ketene } \\
\text { losses) }\end{array}$} \\
\hline & & $\begin{array}{l}\text { CID/OzID } \\
(+/-1.5 \%)\end{array}$ & $\begin{array}{c}\text { LIT MS } \\
\text { (acyl chains) }\end{array}$ & $\begin{array}{l}\text { LIT MS } \\
\text { (ketene } \\
\text { losses) }\end{array}$ & $\begin{array}{c}\text { HCD MS } \\
\text { (acy) } \\
\text { chains) }\end{array}$ & \\
\hline Avanti & 75.8 & 24.2 & 30.7 & 27.7 & 31.3 & 27.6 \\
\hline Chiron & 79.3 & 20.7 & 30.6 & 25.6 & 30.8 & 24.4 \\
\hline Cerilliant & 99.2 & 0.8 & 22.5 & 13.8 & 23.0 & 15.7 \\
\hline
\end{tabular}

